\title{
Semiring with Identity
}

\author{
C. Venkata Lakshmi \\ Dept. of Applied Mathematics, \\ Sri Padmavathi Mahila Visva Vidyalayam, \\ Tirupati - 2 (A.P), India.
}

\author{
T.Vasanthi \\ Dept. of Applied Mathematics, \\ Yogi Vemana University, \\ Kadapa - 516003(A.P), India
}

\begin{abstract}
In this paper, we study the properties of semiring with identity $\mathrm{ab}+\mathrm{a}=\mathrm{a}$ and also study the properties of ordered semiring satisfying the identity $a b+a=a$, for all $a, b$ in $S$. This paper contains mainly two sections. In section 1 , the structure of semirings satisfying the identity $\mathrm{ab}+\mathrm{a}=\mathrm{a}$, for all $\mathrm{a}, \mathrm{b}$ in $\mathrm{S}$ are studied. In section 2 , we characterize totally ordered semirings satisfying the identity $\mathrm{ab}+\mathrm{a}=\mathrm{a}$, for all $\mathrm{a}, \mathrm{b}$ in $\mathrm{S}$.
\end{abstract}

Keywords: Left regular semigroup; PRD; E - inverse semigroup; Quasi separative; Positively totally ordered (p.t.o.); Negatively totally ordered (n.t.o.); Non - negatively ordered; Non - positively totally ordered.

2000 Mathematics Subject Classification: 20M10, $16 Y 60$.

\section{INTRODUCTION}

The study of rings, which are special semirings reveals that multiplicative structure are quite independent of their additive structures though their additive structures are abelian groups. However in semirings it is possible to derive the additive structures from their special multiplicative structures and vice-versa. Semirings have been studied by various researchers in an attempt to broaden techniques coming from the semigroup theory or ring theory or in connection with applications.

The theory of rings and the theory of semigroups have considerable impact on the developments of the theory of semirings and ordered semirings. The works of eminent people like S. Bourne, P. J. Allen, S.K. Iseki , M.P. Grillet, H.E. Stone, H.J. Weinert are to be worth mentioned in the theory of semirings, who use ring theory techniques. J.Hanumanthachari $[3,4]$ andT. Vasanthi have contributed to the theory of semirings using semigroup techniques.

The identity $a b+a=a$ is taken from the book of Jonathan S.Golan[2], entitled "Semirings and their Applications".

In this paper, we investigate the additive and multiplicative properties of semiring satisfying the identity $a b+a=a$

\section{Definition 1.1}

A triple $(\mathrm{S},+, \cdot)$ is said to be a semiring if $\mathrm{S}$ is a non - empty set and ",$+ \bullet$ " are binary operations on $\mathrm{S}$ satisfying that

(i) $(\mathrm{S},+)$ is a semigroup

(ii) $(\mathrm{S}, \bullet)$ is a semigroup

(iii) $\mathrm{a}(\mathrm{b}+\mathrm{c})=\mathrm{ab}+\mathrm{ac}$ and $(\mathrm{b}+\mathrm{c}) \mathrm{a}=\mathrm{ba}+\mathrm{ca}$, for all $\mathrm{a}, \mathrm{b}, \mathrm{c}$ in $\mathrm{S}$.

\section{Example of Semiring 1.2}

Let $S=\{a, b\}$ with the operations given by the following tables:

\begin{tabular}{|c|c|c|}
\hline+ & $\mathrm{a}$ & $\mathrm{b}$ \\
\hline $\mathrm{a}$ & $\mathrm{a}$ & $\mathrm{b}$ \\
\hline $\mathrm{b}$ & $\mathrm{b}$ & $\mathrm{b}$ \\
\hline
\end{tabular}$-$\begin{tabular}{|l|l|l|}
\hline & $\mathrm{a}$ & $\mathrm{b}$ \\
\hline $\mathrm{a}$ & $\mathrm{b}$ & $\mathrm{b}$ \\
\hline $\mathrm{b}$ & $\mathrm{b}$ & $\mathrm{b}$ \\
\hline
\end{tabular}

Then $(\mathrm{S},+, \bullet)$ is a semiring.

\section{Definition 1.3}

A semigroup $(\mathrm{S}, \bullet)$ is said to be

(i) left regular, if it satisfies the identity $a b a=a b$, for all a, $\mathrm{b}, \mathrm{c}$ in $\mathrm{S}$;

(ii) right regular, if it satisfies the identity aba $=\mathrm{ba}$, for all a, $\mathrm{b}, \mathrm{c}$ in $\mathrm{S}$

(iii) regular, if it is both left as well as right regular.

\section{Definition 1.4}

An element 'a' of a multiplicative semigroup ' $\mathrm{S}$ ' is called an $\mathrm{E}$ - inverse if there is an element ' $\mathrm{x}$ ' in $\mathrm{S}$ such that ax (ax) $=$ ax, i.e. $\operatorname{ax} \in \mathrm{E}(\bullet)$, where $\mathrm{E}(\bullet)$ is the set of all multiplicative idempotent elements of $S$.

$\rightarrow$ A Semigroup 'S' is called an E - inverse Semigroup if every element of $\mathrm{S}$ is an $\mathrm{E}$ - inverse.

\section{Definition 1.5}

An element 'a' of an additive semigroup ' $\mathrm{S}$ ' is called an $\mathrm{E}-$ inverse if there is an element ' $x$ ' in $S$ such that $a x+$ $\mathrm{ax}=\mathrm{ax}$, i.e. $\mathrm{ax} \in \mathrm{E}(+)$, where $\mathrm{E}(+)$ is the set of all additive idempotent elements of $\mathrm{S}$.

$\rightarrow$ A Semigroup ' $S$ ' is called an E - inverse Semigroup if every element of $\mathrm{S}$ is an $\mathrm{E}$ - inverse.

Theorem 1.6: Let $(S,+, \bullet)$ be a semiring satisfying the identity $\mathrm{ab}+\mathrm{a}=\mathrm{a}$, for all $\mathrm{a}, \mathrm{b}$ in $\mathrm{S}$. If $(\mathrm{S}, \bullet)$ is a left regular semigroup, then $(\mathrm{S},+)$ is $\mathrm{E}$ - inversive semigroup.

Proof: By hypothesis, $(\mathrm{S}, \bullet)$ is a left regular semigroup i.e., $a b a=a b$

Consider $\mathrm{ab}+\mathrm{a}=\mathrm{a}$, for all $\mathrm{a}, \mathrm{b}$ in $\mathrm{S}$

$\Rightarrow \mathrm{b}(\mathrm{ab}+\mathrm{a})=\mathrm{b} \cdot \mathrm{a}$

$\Rightarrow \mathrm{bab}+\mathrm{ba}=\mathrm{ba}$

$\Rightarrow \mathrm{ba}+\mathrm{ba}=\mathrm{ba}$, for all ba in $\mathrm{E}[+]$

$(\because(\mathrm{S}, \bullet)$ is a left regular, bab $=\mathrm{ba})$

Where $\mathrm{E}[+]$ is the set of all additive idempotents in $(\mathrm{S},+)$

This means that there exists a in $\mathrm{S}$ such that $\mathrm{ba}+\mathrm{ba}=\mathrm{ba}$

$\Rightarrow \mathrm{b}$ is an $\mathrm{E}$ - inversive element

Hence $(S,+)$ is an $\mathrm{E}$ - inversive semigroup.

Definition 1.7

A semigroup $(\mathrm{S}, \cdot)$ is said to satisfy quasi separative if $\quad \mathrm{x}^{2}$ $=\mathrm{xy}=\mathrm{yx}=\mathrm{y}^{2} \Rightarrow \mathrm{x}=\mathrm{y}$, for all $\mathrm{x}, \mathrm{y}$ in $S$. 
Theorem 1.8: Let $(\mathrm{S},+, \cdot)$ be a semiring satisfying the identity $a b+a=a$, for all $a, b$ in $S$. If $S$ contains $a$ multiplicative identity which is also an additive identity, then $(\mathrm{S}, \bullet)$ is quasi separative.

Proof: Let 'e' be the multiplicative identity is also an additive identity

By hypothesis, $a b+a=a$, for all $a, b$ in $S$

To prove that, $(\mathrm{S}, \bullet)$ is quasi separative

i.e., $a^{2}=a b=b a=b^{2} \Rightarrow a=b$, for all $a, b$ in $S$

Let $\mathrm{a}^{2}=\mathrm{ab}$

$\Rightarrow \mathrm{a}^{2}=\mathrm{a} .(\mathrm{b}+\mathrm{e})$

$=\mathrm{ab}+\mathrm{a}$

$$
=\mathrm{a} \quad(\because \mathrm{ab}+\mathrm{a}=\mathrm{a})
$$

$\Rightarrow \mathrm{a}^{2}=\mathrm{a}$

Similarly,

$\Rightarrow b^{2}=$ ba

$$
=\mathrm{b}(\mathrm{a}+\mathrm{e})
$$

$\Rightarrow \mathrm{b}^{2}=\mathrm{ba}+\mathrm{b}$

$$
=\mathrm{b} \quad(\because \mathrm{ba}+\mathrm{b}=\mathrm{b})
$$

$\Rightarrow \mathrm{b}^{2}=\mathrm{b}$

$\therefore \mathrm{a}^{2}=\mathrm{a}$ and $\mathrm{b}^{2}=\mathrm{b}$

If $a^{2}=a b=b a=b^{2}$

$\Rightarrow \mathrm{a}=\mathrm{ab}=\mathrm{ba}=\mathrm{b}$

$\Rightarrow \mathrm{a}=\mathrm{b}$

Hence, $(\mathrm{S}, \bullet)$ is quasi separative.

\section{ORDERING ON SEMIRING SATISFYING THE IDENTITY $\mathbf{a b}+\mathbf{a}=\mathbf{a}$}

The ideas of additive and multiplicative semigroups are taken from the eminent researcher M. Satyanayayana $[1,5]$ paper in the theory of ordered semirings.

In this section, the structure of ordered semirings satisfying the identity $\mathrm{ab}+\mathrm{a}=\mathrm{a}$, for all $\mathrm{a}, \mathrm{b}$ in $\mathrm{S}$ are studied. Here we proved that in a totally ordered PRD semiring $(\mathrm{S},+, \cdot)$ satisfying the identity $a b+a=a$, if $(S,+)$ is p.t.o.(n.t.o.), then 1 is minimum (maximum) element.

\section{Definition 2.1}

A semiring $(\mathrm{S},+, \bullet)$ is said to be totally ordered semiring (t.o.s.r.) if there exists a partially order ' $\leq$ ' on $\mathrm{S}$ such that

(i) $(\mathrm{S},+)$ is a t. o. s. g.

(ii) $(\mathrm{S}, \bullet)$ is a t. o. s. $\mathrm{g}$.

It is usually denoted by $(\mathrm{S},+, \cdot, \leq)$.

Example of totally ordered semirings $\mathbf{2 . 2}$

Consider the set $S=\{1,2,3,4\}$ with the order $1<2<3<4$ and with the following addition and multiplication.

\begin{tabular}{|l|l|l|l|l|}
\hline+ & 1 & 2 & 3 & 4 \\
\hline 1 & 2 & 3 & 4 & 4 \\
\hline 2 & 3 & 4 & 4 & 4 \\
\hline 3 & 4 & 4 & 4 & 4 \\
\hline 4 & 4 & 4 & 4 & 4 \\
\hline
\end{tabular}

\begin{tabular}{|l|l|l|l|l|}
\hline$\cdot$ & 1 & 2 & 3 & 4 \\
\hline 1 & 2 & 4 & 4 & 4 \\
\hline 2 & 4 & 4 & 4 & 4 \\
\hline 3 & 4 & 4 & 4 & 4 \\
\hline 4 & 4 & 4 & 4 & 4 \\
\hline
\end{tabular}

Then $(\mathrm{S},+, \bullet)$ is a totally ordered semiring.

\section{Definition 2.3}

An element $\mathrm{x}$ in a p.o.s.g $(\mathrm{S}, \cdot, \leq)$ is non-negative (nonpositive) if $x^{2} \geq x\left(x^{2} \leq x\right)$.

\section{Definition 2.4}

A p.o.s.g. $(\mathrm{S}, \cdot, \leq)$ is non-negatively (non-positively) ordered if every element of $\mathrm{S}$ is non-negative (non-positive).

\section{Definition 2.5}

An element $\mathrm{x}$ in a p.o.s.g $(\mathrm{S},+, \leq)$ is non-negative (nonpositive) if $\mathrm{x}+\mathrm{x} \geq \mathrm{x}(\mathrm{x}+\mathrm{x} \leq \mathrm{x})$.

\section{Definition 2.6}

A p.o.s.g. $(\mathrm{S},+, \leq)$ is non-negatively (non-positively) ordered if every element of $\mathrm{S}$ is non-negative (non-positive).

\section{Definition 2.7}

A semiring $(\mathrm{S},+, \bullet)$ is said to be a Positive Rational Domain $(\mathrm{PRD})$ if and only if $(\mathrm{S}, \bullet)$ is an abelian group.

Theorem 2.8: Let $(\mathrm{S},+, \bullet)$ be a totally ordered semiring and satisfying the identity $a b+a=a$, for all $a, b$ in $S$. If $(S,+)$ is non - negatively ordered (non - positively ordered.), then (S, •) is non - positively ordered (non - negatively ordered).

Proof: By hypothesis, $a b+a=a$, for all $a, b$ in $S$

Taking $\mathrm{b}=\mathrm{a}$

$\Rightarrow \mathrm{a}^{2}+\mathrm{a}=\mathrm{a}$, for all ' $\mathrm{a}$ ' in $\mathrm{S}$

Suppose $(\mathrm{S},+)$ is non - negatively ordered

$\Rightarrow \mathrm{a}=\mathrm{a}^{2}+\mathrm{a} \geq \mathrm{a}$ and $\mathrm{a}^{2}$

$\Rightarrow \mathrm{a} \geq \mathrm{a}^{2}$

$\therefore(\mathrm{S}, \bullet)$ is non - positively ordered

Suppose $(\mathrm{S},+)$ is non - positively ordered

$\Rightarrow \mathrm{a}=\mathrm{a}^{2}+\mathrm{a} \leq \mathrm{a}$ and $\mathrm{a}^{2}$

$\Rightarrow \mathrm{a} \leq \mathrm{a}^{2}$

$\therefore(\mathrm{S}, \bullet)$ is non - negatively ordered

Theorem 2.9: Let $(\mathrm{S},+, \bullet)$ be a totally ordered $\mathrm{PRD}$ semiring satisfying the identity $a b+a=a$, for all $a, b$ in $S$. If $(S,+)$ is non - negatively ordered (non - positively ordered.), then (S, -) is non - negatively ordered (non - positively ordered).

Proof: Using theorem 1.27, $a+a^{2}=a^{2}$, for all ' $a$ ' in $S$ Since $(S,+)$ is non - negatively ordered, $a^{2}=a+a^{2} \geq a$ $\Rightarrow \mathrm{a}^{2} \geq \mathrm{a}$, for all ' $\mathrm{a}$ ' in $\mathrm{S}$ i,e., $(\mathrm{S}, \bullet)$ is non - negatively ordered Suppose $(\mathrm{S},+)$ is non - positively ordered, $\mathrm{a}^{2}=\mathrm{a}+\mathrm{a}^{2} \leq \mathrm{a}$ $\Rightarrow \mathrm{a}^{2} \leq \mathrm{a}$, for all ' $\mathrm{a}$ ' in $\mathrm{S}$

i,e., $(\mathrm{S}, \bullet)$ is non - positively ordered

\section{Definition 2.10}

In a totally ordered semiring $(\mathrm{S},+, \bullet, \leq)$

(i) $(\mathrm{S},+, \leq)$ is positively totally ordered (p.t.o.), if $\mathrm{a}+\mathrm{b} \geq \mathrm{a}, \mathrm{b}$ for all $\mathrm{a}, \mathrm{b}$ in $\mathrm{S}$ and

(ii) (S, $\bullet, \leq)$ is positively totally ordered (p.t.o.), if $a b \geq a, b$ for all $\mathrm{a}, \mathrm{b}$ in $\mathrm{S}$.

\section{Definition 2.11}

In a totally ordered semiring $(\mathrm{S},+, \cdot, \leq)$

(i) $(\mathrm{S},+, \leq)$ is negatively totally ordered (n.t.o.), if $\mathrm{a}+\mathrm{b} \leq \mathrm{a}, \mathrm{b}$ for all $\mathrm{a}, \mathrm{b}$ in $\mathrm{S}$ and

(ii) (S, $\cdot \leq$ ) is negatively totally ordered (n.t.o.), if $\mathrm{ab} \leq \mathrm{a}, \mathrm{b}$ for all $\mathrm{a}, \mathrm{b}$ in $\mathrm{S}$.

\section{Definition 2.12}

An element $\mathrm{x}$ in a t.o.s. $\mathrm{r}$ is minimal (maximal) if $\mathrm{x} \leq \mathrm{a}$ $(x \geq a)$ for every a in $S$. 
Theorem 2.13: Let $(\mathrm{S},+, \cdot)$ be a totally ordered PRD semiring satisfying the identity $a b+a=a$, for all $a, b$ in $S$. If $(\mathrm{S},+)$ is p.t.o (n.t.o.), then 1 is minimum (maximum) element.

Proof: By theorem 1.27, $1+\mathrm{a}=\mathrm{a}$, for all ' $\mathrm{a}$ ' in $\mathrm{S}$

Suppose $(\mathrm{S},+)$ is p.t.o.

$\Rightarrow \mathrm{a}=1+\mathrm{a} \geq \mathrm{a}$ and 1

$\Rightarrow \mathrm{a} \geq 1$

$\therefore 1$ is the minimum element.

Suppose $(\mathrm{S},+)$ is n.t.o.

$\Rightarrow \mathrm{a}=1+\mathrm{a} \leq \mathrm{a}$ and 1

$\Rightarrow \mathrm{a} \leq 1$

$\therefore 1$ is the maximum element.

\section{CONCLUSION}

(i) The multiplicative structure of the semiring with identity $\mathrm{ab}+\mathrm{a}=\mathrm{a}$ have the additive structure.

(ii) The additive structure of totally ordered semiring satisfying the identity $a b+a=a$, is non-negatively ordered then the multiplicative structure is non-positively ordered. (iii) We introduce PRD in a totally ordered semiring with identity $a b+a=a$ then both the additive and multiplicative structures are having the same ordered.

\section{REFERENCES}

[1] Arif Kaya and M.Satyanarayana, "Semirings satisfying properties of distributive type", Proceeding of the American Mathematical Society, Volume 82, Number 3, July 1981

[2] Jonathan S.Golan, "Semirings and their Applications"

[3] J.Hanumanthachari and D.Umamaheswara Reddy, "A note on maximal and minimal elements in totally ordered semirings". SEA. Bull. Math. Vol. 13.No.2 (1989).

[4] J.Hanumanthachari and K.Venuraju , "The additive semigroup structure of semirings". Mathematics seminar notes, Vol.11(1983),381-386.

[5] M.Satyanarayana - "On the additive semigroup of ordered semirings",Semigroup forum vol.31 (1985), 193199 